ISSN 1980-5098@® DOI: http://dx.doi.org/10.5902/1980509833358

\title{
COMPETIÇÃO VERSUS CRESCIMENTO DE ÁRVORES EM FLORESTA OMBRÓFILA DENSA NO ESTADO DO AMAPÁ - BRASIL
}

\section{COMPETITION VERSUS GROWTH OF TREES IN A RAINFOREST AT AMAPÁ STATE - BRAZIL}

\author{
Silvane Vatraz ${ }^{1}$ José Natalino Macedo Silva ${ }^{2}$ Denis Alder ${ }^{3}$
}

\section{RESUMO}

O objetivo deste trabalho é o de avaliar, comparativamente, diferentes índices de competição independentes da distância (IID) em três dimensões de parcelas, e identificar aqueles que mais se correlacionam com o incremento diamétrico (IPA DAP), indicando o que melhor explica a competição entre as árvores do estrato arbóreo em uma Floresta Ombrófila Densa. Este estudo foi conduzido com dados do Projeto Bom Manejo (Embrapa/CIFOR/ITTO) - Manejo sustentável de florestas de produção em escala comercial na Amazônia brasileira, desenvolvido pela Embrapa Amazônia Oriental, em área da Jari Florestal S.A., município de Vitória do Jari, Estado do Amapá. Foram empregadas três parcelas permanentes de um hectare cada uma (100 $\mathrm{m} \times 100 \mathrm{~m}$ ), nas quais foram medidos todos os indivíduos arbóreos com DAP $\geq 20 \mathrm{~cm}$. Foram comparados os seguintes IID: G (área basal/ha), BAL (Basal Area of Larger Trees), Glover e Hool e Stage. Os índices de competição foram avaliados a partir de análise dos coeficientes de correlação lineares entre cada índice e a variável IPA DAP $\left(\mathrm{cm}^{2}\right.$ ano $\left.^{-1}\right)$. A significância dos coeficientes de correlação foi avaliada pelo $p$ valor $(\alpha=0,05)$. Todas as variações de índices testadas resultaram em correlações de até $20 \%$, consideradas muito fracas. Isto indica que os IID conseguem representar apenas parcialmente a competição em florestas naturais tropicais. No entanto, entre os índices, o índice BAL é o IID que apresentou a maior correlação com o IPA DAP ( $\mathrm{r}=-0,212$ e $p=0,000)$ em parcelas de $50 \mathrm{~m}$ x $50 \mathrm{~m}$ e, portanto, mesmo apresentando uma correlação baixa, é o mais indicado para estudos de competição no crescimento diamétrico em florestas tropicais naturais. Foi detectada uma relação espacial dos IID, em que a correlação diminui com o aumento do tamanho da parcela. Recomendam-se pesquisas adicionais para avaliar os efeitos da competição sobre o crescimento das árvores, bem como o aumento do entendimento de competição intraespecífica e interespecífica, a fim de melhorar a compreensão da dinâmica da competição em florestas tropicais.

Palavras-chave: índices de competição; floresta natural; crescimento em diâmetro; Amazônia.

\begin{abstract}
The objective of this study is to make, comparatively, of different distance independent competition indices (IID) in three dimensions of plots, and to identify those that more correlate with diametric increment (IPA DAP), in order to determine which best explains inter-tree competition in the arboreal stratum in a dense ombrophylous forest. This study was conducted with the Project Good Management data (Embrapa / CIFOR / ITTO) - Sustainable management of production forests on a commercial scale in the Brazilian Amazon, developed by Embrapa Amazônia Oriental in the area of Jari Florestal SA, in the municipality of Vitória do Jari, State of Amapá. Three permanent plots were used of one hectare each $(100 \times 100 \mathrm{~m})$, on which were measured all trees with DBH $\geq 20 \mathrm{~cm}$. The following IIDs were compared: G (basal area/ ha), BAL (Basal Area of Larger Trees), Glover and Hool and Stage. The competition indices were evaluated by analysis of linear correlation coefficients between each index and the PAI DBH $\left(\mathrm{cm}^{-1}\right)$ variable. The significance of

1 Engenheira Florestal, DSc, Professora da Universidade Federal Rural da Amazônia, Instituto de Ciências Agrárias, Av. Perimetral, 2501, CEP 66077-530, Belém (PA), Brasil. vatraz@yahoo.com.br

2 Engenheiro Florestal, PhD, Professor do Programa de Pós-Graduação em Ciências Florestais, Universidade Federal Rural da Amazônia, Av. Perimetral, 2501, Universitário, CEP 66077-530, Belém (PA), Brasil. silvanatalino734@ gmail.com

3 Engenheiro Florestal, PhD, Consulting, Oxford, Reino Unido OX3 8TH, post@denisalder.com
\end{abstract}

Recebido para publicação em 15/09/2015 e aceito em 18/05/2017

Ci. Fl., v. 28, n. 3, jul. - set., 2018 
the correlation coefficients was evaluated by $p$ value $(\alpha=0.05)$. All variations of indexes tested resulted in correlations of up to $20 \%$, considered to be very weak. This indicates that IIDs can represent only partially the competition in tropical natural forests. However, among the indexes, the BAL index is the IID that presented the highest correlation with the PAI DBH $(\mathrm{r}=-0.212$ and $\mathrm{p}=0.000)$ in plots of $50 \mathrm{mx} 50 \mathrm{~m}$ and, therefore, even if it has a low correlation, it is the indicated for competition studies on diametric growth in natural tropical forests. A spatial relationship of the IIDs was detected, where the correlation decreases with the increase of the plot size. Additional research is recommended to evaluate the effects of competition on tree growth, as well to increase understanding of intraspecific and interspecific competition in order to improve knowledge of the competition dynamics in tropical forests.

Keywords: competition indexes, natural forest, diameter growth, Amazônia.

\section{INTRODUÇÃO}

Em florestas naturais tropicais, as árvores estão em contínuo estado de competição, no espaço aéreo pela luz e no solo pela água, minerais e oxigênio. Na medida em que as árvores aumentam de tamanho, suas necessidades individuais também se ampliam (KRAMER; KOZLOWSKI, 1972), e, somados à presença de recursos limitados, contribuem para a diminuição global da taxa de crescimento, do metabolismo e da sobrevivência das árvores (WELDEN; SLAUSON, 1986).

Avaliar a competição de uma floresta é um trabalho difícil, pois muitos fatores influenciam neste processo, entretanto, os índices de competição objetivam expressar o efeito da competição no crescimento das árvores (PEDERSEN et al., 2013). Por isso, a utilização dos índices de competição tornou-se importante parte do manejo florestal em todo o mundo.

Os índices de competição têm sido amplamente empregados na modelagem de crescimento e produção de árvores individuais, assim como na modelagem da dinâmica florestal (PELTONIEMI; MÄKIPÄÄ, 2011). Uma variedade de modelos inclui a competição como um parâmetro, para estimar a mortalidade (EID; TUHUS, 2001; ZHAO et al., 2006), o recrutamento (LEXEROD, 2005), o crescimento diamétrico (ROITMAN, 2011; CUNHA, 2013; VANCLAY et al., 2013; ORELLANA, 2014) e modelos de crescimento em área basal (BOLLANDSAS; NAESSET, 2009; CONTRERAS; AFFLECK; CHUNG, 2011).

Segundo Peng (2000), os índices de competição são separados em dois grupos, o primeiro não considera a posição das árvores dentro da parcela e é chamado de índices independentes da distância (IID) e o segundo, denominado de índices dependentes da distância, leva em consideração a posição relativa entre as árvores e o seu tamanho. Os dois tipos de índices têm sido empregados em florestas com alta diversidade de espécies, no entanto, a literatura não aponta de forma clara que tipo de índice possui maior eficácia (WEISKITTEL et al., 2011). Alguns estudos comparativos concluíram que os independentes da distância são tão eficazes quanto os dependentes da distância (MÄKINEN, 1997).

Entre os índices independentes da distância existem aqueles que são baseados na densidade do povoamento e, portanto, não consideram as dimensões das árvores individualmente; e os baseados em medidas de dominância das árvores, que incluem a dimensão individual da árvore-objetivo e representam sua dominância dentro do povoamento (TOMÉ, 2005).

Como exemplo de índices de competição que se baseiam na densidade do povoamento, cita-se o índice de área basal por hectare (MARTIN; EK, 1984). Ele demonstra a área em metros quadrados por hectare ocupado pelos fustes das árvores que compõem a parcela, sem avaliar a árvore de forma individual. E como exemplo dos que se baseiam na dominância, podem-se citar o índice de BAL (WYKOFF, 1990), que busca quantificar a concorrência por espaço entre as árvores, considerando como competidoras aquelas com área transversal maior que a árvore principal (objetivo); o índice de Glover e Hool (1979), que relaciona a dimensão da árvore-objetivo com a dimensão média das árvores vizinhas, dentro de uma área previamente estabelecida e o índice de Stage (STAGE, 1973), que relaciona a área ocupada $\mathrm{em}^{2}$ da árvore-objetivo com a área ocupada do diâmetro quadrático da parcela.

Deve-se considerar que apesar dos índices independentes da distância não considerarem a distância, sofrem influência do tamanho da parcela e, portanto, são afetados espacialmente (ALDER; SYNNOTT, 1992). Os mesmos autores recomendam o uso de parcelas de 20 × 20 m para o estudo da competição, 
porém, esse tamanho deve ser avaliado e depende da consistência dos dados.

Muitos pesquisadores, como Martins et al. (2011) e Castro et al. (2014), afirmam que a efetividade de um índice de competição é avaliada com o auxílio da ferramenta de correlação linear, relacionando o índice obtido para cada árvore e as variáveis da floresta (incremento em diâmetro, altura e outros).

Neste sentido, o objetivo deste trabalho é o de avaliar índices de competição independentes da distância e verificar aqueles que mais se correlacionam com o incremento diamétrico em $\mathrm{cm} \mathrm{ano}^{-1}$, indicando o que melhor explica a competição entre as árvores do estrato arbóreo em uma Floresta Ombrófila Densa.

\section{METODOLOGIA}

\section{Descrição da área de estudo e dos dados}

O estudo foi conduzido com dados do experimento originalmente denominado Projeto Bom Manejo (Embrapa/CIFOR/ITTO), desenvolvido pela Embrapa Amazônia Oriental. O mesmo está localizado na área florestal da empresa Jari Florestal S. A., na localidade Morro do Felipe, município de Vitória do Jari-AP. Ele possui uma área de 500 ha e foi instalado no ano de 1983, localizado entre as coordenadas geográficas $52^{\circ} 20^{\prime} \mathrm{W}$ de longitude e $0^{\circ} 55^{\prime} \mathrm{S}$ de latitude, com uma altitude de aproximadamente $150 \mathrm{~m}$ (YARED et al., 2000). O monitoramento da floresta ocorreu entre os anos de 1984 a 2011, ocorrendo medições em oito ocasiões: 1984, 1986, 1988, 1990, 1994, 1996, 2004 e 2011. No ano de 1985, houve uma colheita, na qual foram reduzidos cerca de $15 \%$ do volume comercial das árvores com DAP $\geq 60 \mathrm{~cm}$. Foram exploradas cerca de três árvores por hectare e o volume correspondeu a $26 \mathrm{~m}^{3}$ ha-1.

Neste experimento foram instaladas parcelas permanentes quadradas, medindo $100 \mathrm{~m} \times 100 \mathrm{~m}$ (1 ha), nas quais foram medidos todos os indivíduos arbóreos com DAP $\geq 20 \mathrm{~cm}$ (AZEVEDO et al., 2012). Para a medição das parcelas permanentes empregou-se a metodologia sugerida por Silva e Lopes (1984). As informações e variáveis utilizadas neste estudo foram: número da árvore; classe de identificação do fuste (CIF), para selecionar as árvores vivas; diâmetro a altura do peito (DAP) e identificação botânica.

O clima na região da área de estudo é do tipo Ami (GOMIDE, 1997) e a precipitação média anual é de $2.234 \mathrm{~mm}$ (AZEVEDO et al., 2012). A vegetação é do tipo Floresta Tropical de Terra Firme ou Floresta Ombrófila Densa (VELOSO; RANGEL-FILHO; LIMA, 1991). E os solos que predominam nesta área são variados, como: Argissolo Vermelho-Amarelo, Latossolo Amarelo, Latossolo Vermelho-Amarelo, Latossolo Vermelho-Escuro e Neossolo Litólico (EMPRESA DE PESQUISA ENERGÉTICA, 2010).

\section{Índices de competição independentes da distância}

Neste trabalho, toda árvore que possui sua competição avaliada de forma individual, é considerada "árvore-objetivo", de acordo com os diferentes índices de competição. E, como todas as árvores das parcelas foram avaliadas, todas as árvores serão em um dado momento "árvores-objetivo" (VATRAZ; ALDER; SILVA, 2016). Os índices de competição avaliados e comparados estão descritos na Tabela 1: 
TABELA 1: Índices de competição independentes da distância avaliados neste trabalho.

TABLE 1: Distance independent competition indices in this work.

\begin{tabular}{lcc}
\hline \multicolumn{1}{c}{ Índice } & Fórmula & Autores/Fonte \\
\hline Área basal & $G=\sum_{i=1}^{n i} g_{i}$ & Martin e Ek (1984) \\
BAL (Basal Area of Larger Trees) & $B A L=\sum_{i=1}^{n} g m$ & Wykoff (1990) \\
Glover e Hool & $I_{G H}=\frac{d_{i}^{2}}{\bar{d}^{2}}$ & Glover e Hool (1979) \\
Stage & $I C_{S T A G E}=\frac{g_{i}}{g_{q}}$ & Stage (1973) \\
\hline
\end{tabular}

Em que: $n=$ número de árvores com DAP maior que a árvore-objetivo; $g m=$ área transversal $\left(\mathrm{m}^{2}\right)$ das árvores com DAP maiores que a árvore-objetivo; $n_{i}=$ número de árvores da parcela; $g=$ área transversal $\left(\mathrm{m}^{2}\right) ; d_{i}^{2}=$ diâmetro da árvore-objetivo $(\mathrm{cm})$ elevado ao quadrado; $\bar{d}^{2}=$ diâmetro médio do povoamento $(\mathrm{cm})$ elevado ao quadrado; $g_{i}=$ área transversal da árvore-objetivo $\left(\mathrm{m}^{2}\right)$ e $g_{q}=$ área transversal da árvore média $\left(\mathrm{m}^{2}\right)$ (definida pelo diâmetro quadrático).

No caso específico do índice de área basal, o valor calculado é por parcela e desta forma, um mesmo valor de $\mathrm{G}$ é atribuído a cada árvore individual da mesma parcela.

\section{Cálculo do incremento diamétrico}

O incremento periódico anual em diâmetro (IPA DAP) foi obtido pela diferença entre as medidas de diâmetro das árvores com DAP $\geq 20 \mathrm{~cm}$, no período de 1984 e 2004, empregando a seguinte fórmula: $\left(\mathrm{DAP}_{2004}-\mathrm{DAP}_{1984}\right) / \mathrm{T}$, em que $\mathrm{T}$ refere-se ao tempo, em anos decorridos entre as duas medições.

\section{Tamanho da parcela}

Como os índices independentes da distância possuem relação espacial, foram testados três tamanhos de parcelas. Para operacionalizar essa avaliação, os índices foram calculados para o tamanho original das parcelas, ou seja, $100 \mathrm{~m} \times 100 \mathrm{~m}$ (1 ha) e depois subdivididas em dois tamanhos, $50 \mathrm{~m} \times 50 \mathrm{~m}(0,25 \mathrm{ha}) \mathrm{e}$ $20 \mathrm{~m} \times 20 \mathrm{~m}(0,04 \mathrm{ha})$.

\section{Avaliação dos índices de competição}

A correlação linear de Pearson (r) foi empregada para avaliar a correlação entre os índices calculados para cada árvore e a variável IPA DAP em $\mathrm{cm} \mathrm{ano}^{-1}$. O coeficiente de correlação (r) pode variar de -1 a 1 e, quando se tem um "r" próximo de 0 não é uma evidência de que não exista uma relação, mas apenas de que se existe tal relação, ela pode não ser linear (BUSSAB; MORETTIN, 2013). O valor de $p$ foi utilizado para avaliar a significância dos coeficientes, em que, se o valor de $p$ calculado for menor ou igual ao nível de significância $(\alpha<0,05)$, infere-se que existe correlação linear e se for maior, não existe correlação (BUSSAB; MORETTIN, 2013).

\section{RESULTADOS E DISCUSSÃO}

Foram avaliadas 394 árvores, distribuídas em 42 famílias, 89 gêneros e 94 espécies, nas medições de 1984 e 2004.

Uma análise prévia dos dados pode ser visualizada na Figura 1, na qual se podem observar os efeitos da competição no crescimento das árvores, à medida que se nota um maior incremento diamétrico $\left(\mathrm{cm} \mathrm{ano}{ }^{-1}\right)$ nas árvores de maior diâmetro. 


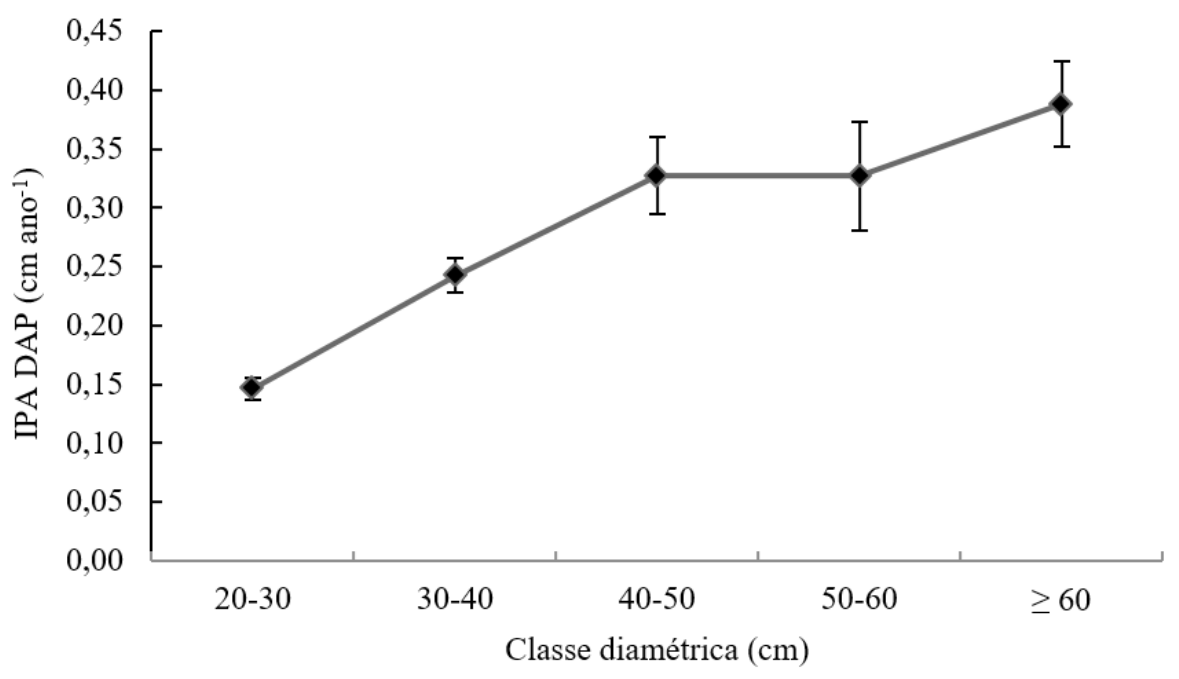

FIGURA 1: Incremento periódico anual em diâmetro das árvores com DAP $\geq 20 \mathrm{~cm}$, por classe diamétrica, avaliada no período 1984 e 2004, em uma Floresta Ombrófila Densa, localizada em Vitória do Jari-AP (As barras representam o erro padrão).

FIGURE 1: Periodic annual increment in diameter of trees with $\mathrm{DBH} \geq 20 \mathrm{~cm}$ by diametric class, evaluated in the period 1984 and 2004, in a dense ombrophylous forest, located in Vitoria do Jari-AP (The bars represent the standard error).

Esse efeito pode ser explicado pelo fato das árvores de maior porte estarem localizadas no estrato superior da floresta e apresentarem menor competição (CUNHA, 2013). Desta maneira, por apresentarem melhores condições de iluminação de suas copas, também apresentam maiores taxas de crescimento (LIEBERMAN; PUTZ, 1987; KING; MAINDONALD, 1999; COSTA; SILVA; CARVALHO, 2008). Por conseguinte, árvores menores, localizadas no sub-bosque, apresentam maior competição e menores taxas de crescimento (CARVALHO, 1992) e maiores probabilidades de mortalidade (CASTRO et al., 2014).

\section{Correlações}

As correlações lineares entre os índices de competição independentes da distância com a variável da floresta testada em cada tamanho de parcela podem ser observadas na tabela abaixo (Tabela 2).

TABELA 2: Correlações lineares entre os índices independentes (IID) e a variável IPA DAP ( $\left.\mathrm{cm} \mathrm{ano}^{-1}\right)$ em árvores individuais do estrato arbóreo em Floresta Ombrófila Densa, localizada em Vitória do Jari-AP.

TABLE 2: Linear correlations among independent indices (IID) and PAI DBH $\left(\mathrm{cm} \mathrm{ano}^{-1}\right)$ in individual trees of the arboreal stratum in a dense ombrophylous forest, located in the municipality of Vitória do Jari-AP.

\begin{tabular}{cccc}
\hline \multirow{2}{*}{ Índice } & \multirow{2}{*}{ Área de competição } & \multicolumn{2}{c}{$\operatorname{IPA}$ DAP $\left(\mathrm{cm} \mathrm{ano}^{-1}\right)$} \\
\cline { 3 - 4 } & Parcelas de $20 \mathrm{~m} \times 20 \mathrm{~m}$ & $-0,074$ & 0,145 \\
\hline $\mathrm{G}$ & & $-0,090$ & 0,075 \\
BAL & & 0,178 & 0,000 \\
Glover e Hool & & 0,184 & 0,000 \\
Stage & & $-0,104$ & 0,039 \\
\hline G & Parcelas de $50 \mathrm{~m} \times 50 \mathrm{~m}$ & $-0,212$ & 0,000 \\
BAL & & \multicolumn{3}{c}{ Continuação... }
\end{tabular}


TABELA 2: Continuação...

TABLE 2: Continued..

\begin{tabular}{cccc}
\hline \multirow{2}{*}{ Índice } & \multirow{2}{*}{ Área de competição } & \multicolumn{2}{c}{$\operatorname{IPA}$ DAP $\left(\mathrm{cm} \mathrm{ano}^{-1}\right)$} \\
\cline { 3 - 4 } & & $\mathrm{r}$ & $p$-valor \\
\hline Stage & Parcelas de $100 \mathrm{~m} \times 100 \mathrm{~m}$ & 0,003 \\
\hline $\mathrm{G}$ & & $-0,090$ & 0,074 \\
BAL & $-0,160$ & 0,001 \\
Glover e Hool & 0,101 & 0,044 \\
Stage & 0,102 & 0,044 \\
\hline
\end{tabular}

Se $p$-valor for menor ou igual ao nível de significância $\alpha<0,05$, os resultados são significativos estatisticamente, caso contrário, serão não significativos.

Em relação às interações dos IID com o IPA DAP, as correlações significativas diminuíram com o aumento do tamanho das parcelas para os índices de Glover Hool e Stage. Para o índice BAL, ocorreu resultado semelhante, no entanto, entre as parcelas de $20 \mathrm{~m}$ x $20 \mathrm{~m}$ e $50 \mathrm{~m}$ x $50 \mathrm{~m}$, a correlação aumentou, porém, a correlação da parcela menor ( 20 m x 20 m) não foi significativa estatisticamente. Portanto, pode-se considerar que a correlação entre o índice BAL e o IPA DAP também diminuiu com o aumento do tamanho das parcelas. As correlações dos IID com o IPA DAP $\left(\mathrm{cm} \mathrm{ano}^{-1}\right)$ apresentaram correlações que variam de $\mathrm{r}$ $=-0,074$ a r $=-0,212$ e $\mathrm{r}=0,101 \mathrm{ar}=0,184$, todavia, considerando apenas os resultados com significância estatística, tem-se: $r=-0,104$ a r $=-0,212$ e $r=0,101$ a $r=0,184$.

Avaliando as correlações em módulo, isto é, sem considerar resultados com sinais positivos ou negativos, o índice de BAL apresentou a correlação mais alta, contudo, fraca, com $r=-0,212$ na parcela de $50 \mathrm{~m} \times 50 \mathrm{~m}$. E continuou a apresentar uma correlação fraca para a parcela de $100 \mathrm{~m}$ x $100 \mathrm{~m}$, porém, com correlação menor, de $\mathrm{r}=-0,160$. O índice de Stage apresentou a segunda maior correlação com o IPA DAP $(\mathrm{r}=0,184)$, todavia, em uma parcela menor $(20 \mathrm{~m}$ x $20 \mathrm{~m})$ do que a maior correlação de BAL. O índice de Glover e Hool apresentou um resultado muito semelhante ao índice de Stage, revelando a sua maior correlação com um $\mathrm{r}=0,178$ também para a parcela de $20 \mathrm{~m}$ x $20 \mathrm{~m}$. O índice de área basal (G) apresentou apenas uma correlação significativa estatisticamente, que foi considerada fraca com $r=-0,104$, para a parcela de $50 \mathrm{~m} \times 50 \mathrm{~m}$.

\section{Avaliação dos índices}

O índice de BAL apresentou valores de correlações negativos, indicando que o IPA DAP se opõe aos valores do referido índice. Esta correlação negativa do índice de BAL também mostra que maiores valores do índice correspondem a árvores de menor diâmetro. Esse fato também foi observado por Castro et al. (2014) e explicado por Martins et al. (2011), pois é uma situação relacionada com a própria fórmula do índice de BAL, que consiste no somatório das áreas transversais das árvores que apresentam diâmetros imediatamente maiores do que a árvore-objetivo, de forma que árvores com menores diâmetros apresentam maiores valores no referido índice.

O inverso ocorre com os índices de Glover e Hool e de Stage, que apresentam menores índices para as árvores que sofrem maior competição (TOMÉ; BURKHART, 1989; MARTINS et al., 2011; CASTRO et al., 2014). Fato também explicado pela fórmula, em que estes índices são derivados da razão entre uma variável dendrométrica da árvore-objetivo em relação à média da mesma variável para o povoamento, que consideram as dimensões de suas vizinhas. Isto quer dizer que, árvores-objetivo que apresentem sua variável dendrométrica inferior à média de suas vizinhas, apresentarão menores índices.

Uma fragilidade dos IID aqui testados deve ser levada em consideração quando no uso dos mesmos, pois se deve lembrar, que não se pode apenas considerar que somente árvores de maior porte podem exercer pressão de competição sobre as outras e por isso estes índices devem ser empregados com cautela. Outra questão apontada por Castro et al. (2014) é o fato de que os índices de competição são calculados de acordo 
com os tamanhos dos fustes das árvores e, desta forma, para fustes de mesmo porte são atribuídos índices de competição de valores muito semelhantes, mesmo que não possuam a mesma taxa de crescimento.

O uso de variáveis do povoamento para descrever o crescimento de árvores individuais é um dos principais problemas dos IID em florestas naturais, pois, nesse método, todas as árvores que abrangem a parcela contribuem igualmente para a estimativa da competição, pois é assumido que o espaço é igual ao longo de cada árvore-objetivo, e que esse espaço seria adequado para descrever a competição entre as árvores (MÄKINEN, 1997).

De maneira geral, em relação ao tamanho das parcelas, a maior correlação com o IPA DAP ocorreu com o índice de BAL, em parcelas com largura de 50 metros. Esta informação corrobora com os estudos de Roitman (2011) em uma floresta de galeria, na qual sua pesquisa sugere a utilização de parcelas com pelo menos $40 \mathrm{~m}$ de largura para o estudo de índices de competição. Orellana (2014) também empregou parcelas de $50 \mathrm{~m}$ x $50 \mathrm{~m}$ em sua pesquisa em Floresta Ombrófila Mista, no sul do Brasil.

A pesquisa de Castro et al. (2014) em uma Floresta Estacional Semidecidual Montana, também apontou para resultados de correlações fracas, abaixo de 30\%, entre o índice de competição e o incremento diamétrico. O índice de Glover e Hool apresentou a maior correlação para esta variável $(\mathrm{r}=0,26)$, seguida do índice de Stage $(r=0,17)$ e do índice de BAL $(r=-0,20)$. Resultados muito semelhantes ao do presente estudo, sendo apenas discretamente diferente para o índice de Glover e Hool.

Monserub e Sterba (1999) e Eid e Tuhus (2001) salientaram que o índice de BAL seria um excelente índice de competição. Outros autores, como Martins et al. (2011) recomendaram o uso do índice de Stage, que também não necessita de informações espaciais, assim como o BAL, e apresenta um bom desempenho na avaliação da competição além de representar certo realismo biológico. E, portanto, pode-se dizer que a utilização do índice de BAL $(\mathrm{r}=-0,212)$ ou de Stage $(\mathrm{r}=0,151)$ pode ser empregada na avaliação da competição.

Em florestas plantadas, os IID apresentam melhores resultados, como é o caso de plantios comerciais de eucalipto, cuja correlação com o IPA DAP variou entre $r=-0,74$ e 0,52 (MARTINS et al., 2011); para Pinus taeda, as correlações encontradas foram de $\mathrm{r}=0,23$ a 0,31 entre estas mesmas variáveis (DANIELS; BURKHART; CLASON, 1986). É provável que nesse tipo de floresta, em que se tem apenas uma espécie, plantada a um espaçamento previamente planejado, com a mesma idade, o vigor de crescimento das árvores seja mais homogêneo e, portanto, os IID detectariam com maior sensibilidade a competição existente.

Em florestas tropicais existem grandes variações no crescimento (altura, diâmetro) em indivíduos do mesmo tamanho. Enquanto algumas espécies crescem muito em um período, outras não crescem. Essa heterogeneidade de espécies na floresta levam às baixas correlações com a variável IPA DAP (CASTRO et al., 2014), constatação também relatada por Cunha (2013).

Tomé e Burkhart (1989) relatam que mesmo com correlações baixas entre os índices de competição e o incremento em diâmetro, ainda assim, os índices de competição contribuem de forma significativa nos modelos de crescimento.

Resultados não tão otimistas, também foram relatados por Pukkala e Kolstrem (1987), os autores mencionam que os melhores índices de competição poderiam explicar somente até $50 \%$ do crescimento diamétrico. Porém, os resultados encontrados neste presente estudo não passam de $20 \%$, portanto, muito abaixo da referência de Pukkala e Kolstrem (1987), contudo, dentro do esperado pela sua pesquisa.

Conforme mostrou a pesquisa de Zhao et al. (2006), os efeitos da competição no crescimento das árvores são muito fracos para serem detectados em florestas naturais ricas em espécies. Isso é corroborado pelos resultados alcançados neste estudo, em que as correlações são baixas com o incremento em diâmetro.

As diferentes e simultâneas interações que todas as espécies participam uma com outras árvores, deve ser um fator a ser considerado na justificativa de correlações baixas em florestas tropicais. Uma é a interação por interferência, que é física e direta, e acontece quando um indivíduo impede de forma direta outros organismos de explorarem um recurso (BEGON; HARPER; TOWNSEND; 1996), seus efeitos geralmente são negativos e podem ser de grande impacto (MILLER, 1994). A outra é a competição por exploração, que ocorre quando uma espécie explora um recurso em comum com outra, mas sem contato direto com aquela espécie (ODUM; BARRET, 2007), seus efeitos podem ser positivos ou negativos (MILLER, 1994). Assim, os efeitos competitivos líquidos em florestas tropicais, podem se apresentar baixos e fracos, pois os efeitos negativos inseridos pela competição por interferência direta podem ser minimizados por algum fator que 
facilite efeitos positivos na competição indireta (por exploração), por exemplo, maior disponibilidade de recursos, como água e nutrientes (MILLER, 1994).

Segundo Castro et al. (2014), a complexidade de se medir e interpretar as interações bióticas, tendo em vista expandir o entendimento da competição em florestas tropicais, propõe-se a desenvolver índices de competição com distintas abordagens, que considerem os conceitos de competição intraespecífica e interespecífica. Outros autores, como Uriarte et al. (2004), também afirmam a importância da competição interespecífica em florestas tropicais.

Desta forma, o estudo empírico de IID na floresta estudada resultou em correlações muito fracas, quando avaliado o incremento em diâmetro. Isto indica que os índices parecem apenas colaborar parcialmente na descrição da interferência da competição no crescimento das árvores, pois, para que isso fosse operacionalizado, seria necessário incluir toda a diversidade genética das espécies e identificar padrões de crescimento para cada uma delas e entre elas e ainda considerar todas as relações com diferentes áreas de competição (tamanho de parcela), bem como, identificar a competição intraespecífica e interespecífica das árvores.

Desta forma, o desenvolvimento de índices de competição eficazes para a aplicabilidade em florestas tropicais constitui um desafio ao crescente interesse à ampliação do conhecimento da competição, a fim de melhorar a compreensão da dinâmica da competição.

\section{CONCLUSÕES}

Pode-se constatar que:

Entre os IID, o que expressou a maior correlação foi o índice BAL $(\mathrm{r}=-0,212$ e $p=0,000)$. Este foi considerado o mais indicado para o estudo de correlações com o IPA DAP $\left(\mathrm{cm} \mathrm{ano}^{-1}\right)$ na floresta estudada, apesar de apresentar uma correlação considerada fraca.

$\checkmark$ Evidenciou-se a relação espacial dos IID, pois as correlações diminuíram com o aumento do tamanho das parcelas. A maior correlação ocorreu na parcela de $50 \mathrm{~m}$ x $50 \mathrm{~m}$, com o uso do índice BAL.

$\checkmark$ Todas as variações de índices de competição aqui testadas resultaram em correlações com o IPA DAP muito fracas (10 a 20\%). Isto indica que os índices conseguem representar parcialmente a competição em florestas tropicais naturais.

\section{AGRADECIMENTOS}

Os autores agradecem a Embrapa Amazônia Oriental, especialmente ao pesquisador Ademir Roberto Ruschel, pela disponibilização do banco de dados do experimento originalmente denominado Projeto Bom Manejo (Embrapa/CIFOR/ITTO), para a realização deste estudo, oriundo do doutoramento da primeira autora.

\section{REFERÊNCIAS}

ALDER, D.; SYNNOTT, T. J. Permanent sample plot techniques for mixed tropical forest. Oxford: Forestry Institute, 1992. 124 p. (Tropical Forestry Paper, 25).

AZEVEDO, C. P. et al. Eficiência de tratamentos silviculturais por anelamento na floresta do Jari, Amapá. Floresta, Curitiba, v. 42, n. 2, p. 315-324, set. 2012.

BEGON, M.; HARPER, J. L.; TOWNSEND, C. R. Ecology: individuals, populations and communities. 2nd ed. Oxford: Blackwell Science, 1996. 1068 p.

BOLLANDSAS, O. M.; NAESSET, E. Weibull models for single-tree increment of Norway spruce, Scots pine, birch and other broadleaves in Norway. Scandinavian Journal of Forest Research, Uppsala, v. 24, p. 54-66, mar. 2009.

BUSSAB, W. O.; MORETTIN, P. A. Estatística básica. 8. ed. São Paulo: Saraiva, 2013.

CARVALHO, J. O. P. Structure and dynamics of logged over Brazilian Amazonian rain forest. 1992. 215 f. Tese (Doutorado em Ciências Florestais) - University of Oxford, Oxford, 1992. 
CASTRO, R. et al. Competição em nível de árvore individual em uma floresta estacional semidecidual. Silva Lusitana, Lisboa, v. 22, n. 1, p. 43-66, mar. 2014.

CONTRERAS, M. A.; AFFLECK, D.; CHUNG, W. Evaluating tree competition indices as predictors of basal area increment in western Montana forests. Forest Ecology and Management, Amsterdam, v. 262, p. 1939-1949, dez. 2011.

COSTA, D. H. M.; SILVA, J. N. M.; CARVALHO, J. O. P. Crescimento de árvores em uma área de terra firme na floresta nacional do Tapajós após a colheita de madeira. Revista de Ciências Agrárias, Belém, n. 50, p. 63-76, jul./dez. 2008.

CUNHA, T. A. Competição assimétrica e o incremento diamétrico de árvores individuais de Cedrella odorata L. na Amazônia ocidental. Acta Amazonica, Manaus, v. 43, n. 1, p. 9-18, jan. 2013.

DANIELS, R. F.; BURKHART H. E.; CLASON T. R. A comparison of competition measures for predicting growth of loblolly pine trees. Canadian Journal of Forest Research, Ottawa, v. 16, n. 6, p. 1230-1237, jun. 1986.

EMPRESA DE PESQUISA ENERGÉTICA. Bacia hidrográfica do Rio Jari/ PA-AP: estudos de inventário hidrelétrico. Relatório final, Apêndice D, 2010. Disponível em: <http://www.epe.gov.br/MeioAmbiente/ Documents/AAI\%20Jari/10\%20-\%20Invent\%C3\%A1 rio\%20Hidrel\%C3\%A9trico\%20Jari\%20-\%20 Diagn\%C3\%B3stico\%20Socioambiental\%20-\%20Tomo\%20II.pdf $>$. Acesso em: 9 mar. 2015.

EID, T., TUHUS, E. Models for individual tree mortality in Norway. Forest Ecology and Management, Amsterdam, v. 154, p. 69-84, nov. 2001.

GOMIDE, G. L. A. Estrutura e dinâmica de crescimento de florestas tropicais primárias e secundária no estado do Amapá. 1997. 179 f. Dissertação (Mestrado em Engenharia Florestal) Universidade Federal do Paraná, Curitiba, 1997.

GLOVER G. R.; HOOL, J. N. A basal area ratio predictor of loblolly pine plantation mortality. Forest Science, Bethesda, v. 25, p. 275-282, jun. 1979.

KING, D. A.; MAINDONALD, J. H. Tree architecture in relation to leaf dimensions and tree stature in temperate and tropical rain forests. Journal of Ecology, London, v. 87, p. 1012-1024, dez. 1999.

KRAMER, P. J.; KOZLOWSKI, T. T. Fisiologia das árvores. Lisboa: Fundação Calouste Gulbekian, 1972. LEXEROD, N. L. Recruitment models for different tree species in Norway. Forest Ecology and Management, Amsterdam, v. 206, p. 91-108, fev. 2005.

LIEBERMAN, D.; PUTZ, F. E. The dynamics of tree populations in tropical forest: a review. Journal of Tropical Ecology, Winchelsea, n. 3, p. 359-366, ago. 1987.

MÄKINEN, H. Possibilities of competition indices to describe competitive differences between scots pines families. Silva Fennica, Vantaa, v. 31, n. 1, p. 43-52, jan. 1997.

MARTIN G. L.; EK, A. R. A comparison of competition measures and growth models for predicting plantation red pine diameter and height growth. Forest Science, Bethesda, v. 30, p. 731-743, set. 1984.

MARTINS, F. B. et al. Índices de competição em árvores individuais de eucalipto. Pesquisa Agropecuária Brasileira, Brasília, v. 46, n. 9, p. 1089-1098, set. 2011.

MONSERUD, R. A.; STERBA, H. A. A basal area increment model for individual trees growing in even and uneven-aged forests stands in Austria. Forest Ecology and Management, Amsterdam, v. 80 , n. 1 , p. $57-80$, jan. 1996.

MILLER, T. E. Direct and indirect species interactions in an early old-field plant community. American Naturalist, Chicago, v. 143, p. 1007-1025, 1994.

ODUM, E. P.; BARRETT, G. W. Fundamentos da ecologia. São Paulo: Thompson Learning, 2007. 612 p. ORELLANA, E. Sucessão florestal, regimes de manejo e competição avaliados por modelos de árvore individual em um fragmento de Floresta de Araucária. 2014. 154 f. Tese (Doutorado em Engenharia Florestal) - Universidade Federal do Paraná, Curitiba, 2014.

PEDERSEN, R. O. et al. On the evaluation of competition indices: the problem of overlapping samples. Forest Ecology and Management, Amsterdam, n. 310, p. 120-133, dez. 2013.

PELTONIEMI, M.; MÄKIPÄÄ, R. Quantifying distance-independent tree competition for predicting Norway spruce mortality in unmanaged forests. Forest Ecology and Management, Amsterdam, v. 261, p. 30-42, jan. 2011.

PENG, C. Growth and yield models for uneven-aged stands: past, present and future. Forest Ecology and 
Management, Amsterdam, v. 132, p. 259-279, nov. 2000.

PUKKALA, T.; KOLSTREM, T. Competition indices and the prediction of radial growth in Scots pine. Silva Fennica, Vantaa, v. 21, p. 55-67, jan. 1987.

ROITMAN, I. Modelagem espacial e temporal na mata de galeria do gama- DF. 2011. $169 \mathrm{f}$. Tese (Doutorado em Ecologia) - Universidade Federal de Brasília, Brasília, 2011.

SILVA, J. N. M.; LOPES, J. C. A. Inventário florestal contínuo em florestas tropicais: a metodologia utilizada pela EMBRAPA-CPATU na Amazônia brasileira. Belém: Embrapa; CPATU, 1984. 36 p.

STAGE, A. R. Prognosis model for stand development. Washington: [s. n.], 1973. 32 p.

TOMÉ, M. Modelação do crescimento e da produção de povoamentos florestais. Lisboa: Universidade Técnica de Lisboa, 2005. 52 p. (Textos didáticos do GIMREF).

TOMÉ, M.; BURKHART, H. E. Distance-dependent competition measures for predicting growth of individual trees. Forest Science, Bethesda, v. 35, p. 816-831, set. 1989.

URIARTE, M. et al. A spatially explicit model of sapling growth in a tropical forest: does the identity of neighbours matter? Journal of Ecology, London, v. 92, p. 348-360, abr. 2004.

VANCLAY, J. K. et al. Spatially-explicit competition in a mixed planting of Araucaria cunninghamii and Flindersia braleyana. Annals of Forest Science, Champenoux, v. 70, n. 6, p. 611-619, 2013.

VATRAZ, S.; ALDER, D.; SILVA, J. N. M. Índices de competição dependentes da distância do estrato arbóreo na Amazônia brasileira. Espacios, Caracas, v. 37, n. 27, p. 3, 2016.

VELOSO, H. P.; RANGEL FILHO, A. L. R.; LIMA, J. C. A. Classificação da vegetação brasileira adaptada a um sistema universal. Rio de Janeiro: IBGE, 1991. 124 p.

YARED, J. A. G. et al. Contribuições do Projeto Silvicultura Tropical: cooperação internacional Brasil / Reino Unido. Belém: Embrapa Amazônia Oriental; DFID, 2000. 28 p. (Documentos, 52).

WEISKITTEL, A. R. et al. Forest Growth and Yield Modeling. Chichester: Wiley-Blackwell, 2011.

WELDEN, C. W.; SLAUSON, W. L. The intensity of competition versus its importance: an overlooked distinction and some implications. Quarterly Review of Biology, Stony Brook, v. 61, p. 23-44, may 1986. WYKOFF, W. R. A basal area increment model for individual Conifers in the Northern Rocky Mountains. Forest Science, Bethesda, v. 36, p. 1077-1104, dez. 1990.

ZHAO, D. et al. Modeling neighborhood effects on the growth and survival of individual trees in a natural temperate species rich forest. Ecological Modelling, Amsterdam, v. 196, n. 1, p. 90-102, jul. 2006. 